\title{
ARTICLE OPEN Decoherence benchmarking of superconducting qubits
}

\author{
Jonathan J. Burnett $\mathbb{D}^{1,2}$, Andreas Bengtsson $\mathbb{D}^{1}$, Marco Scigliuzzo ${ }^{1}$, David Niepce $\mathbb{D}^{1}$, Marina Kudra ${ }^{1}$, Per Delsing $\mathbb{D}^{1}$ and \\ Jonas Bylander (iD
}

We benchmark the decoherence of superconducting transmon qubits to examine the temporal stability of energy relaxation, dephasing, and qubit transition frequency. By collecting statistics during measurements spanning multiple days, we find the mean parameters $\overline{T_{1}}=49 \mu \mathrm{s}$ and $\overline{T_{2}^{*}}=95 \mu \mathrm{s}$; however, both of these quantities fluctuate, explaining the need for frequent re-calibration in qubit setups. Our main finding is that fluctuations in qubit relaxation are local to the qubit and are caused by instabilities of nearresonant two-level-systems (TLS). Through statistical analysis, we determine sub-millihertz switching rates of these TLS and observe the coherent coupling between an individual TLS and a transmon qubit. Finally, we find evidence that the qubit's frequency stability produces a $0.8 \mathrm{~ms}$ limit on the pure dephasing which we also observe. These findings raise the need for performing qubit metrology to examine the reproducibility of qubit parameters, where these fluctuations could affect qubit gate fidelity.

npj Quantum Information (2019)5:54; https://doi.org/10.1038/s41534-019-0168-5

\section{INTRODUCTION}

Universal, fault-tolerant quantum computers-a Holy Grail of quantum information processing - are currently being pursued by academia and industry alike. To achieve fault tolerance in a quantum information processor, a scheme for quantum error correction $^{1}$ is needed due to the limited coherence lifetimes of its constituent qubits and the consequently imperfect quantum-gate fidelities. Such schemes, e.g., the surface code, ${ }^{2}$ rely on gate fidelities exceeding a certain break-even threshold. Adequately high fidelity was recently demonstrated with superconducting qubits; $^{3}$ however, this break-even represents a best-case scenario without any temporal or device-to-device variation in the coherence times or gate fidelities. Therefore, a fault-tolerant quantum computer importantly requires not only improvements of the best-case single- ${ }^{4}$ and two-qubit ${ }^{3}$ gate fidelities: it actually requires the typical performance-in the presence of fluctuations - to exceed the error correction threshold. In the more immediate term, so-called Noisy Intermediate-Scale Quantum (NISQ) ${ }^{5}$ circuits will be operated without quantum error correction. In NISQ systems, gate fidelities and the fluctuations thereof directly limit the circuit depth, i.e., the number of consecutive gates in an algorithm that can be successfully implemented.

In experiments with superconducting qubits, it is usual to perform qubit metrology ${ }^{6}$ to benchmark the gate fidelity and quantify its error, although these benchmarks are not typically repeated in time to determine any temporal dependence. Since gate fidelities are at least partially limited by qubit $T_{1}$ energy relaxation, ${ }^{4}$ one would expect a fluctuation in gate fidelity resulting from a fluctuation in the underlying decoherence parameters. However, benchmarking of decoherence, to quantify the mean lifetime together with its stability or variation, is also uncommon. Consequently, it is unclear whether reports on improvements in coherence times-cf. the review by Oliver and Welander $^{7}$ and that by Gu and Frisk Kockum et al. ${ }^{8}$ - are reports of typical or of exceptional performance. Quantifying this difference is crucial for future work aimed at improving qubit coherence times.

In this paper, we benchmark the stability of decoherence properties of superconducting qubits: $T_{1}, T_{2}^{*}$ (free-induction decay), $T_{\phi}$ (pure dephasing), and $f_{01}$ (qubit frequency). This study is distinct from numerous studies that report on singular measurements of qubit lifetimes for different background conditions, such as temperature ${ }^{9}$ or magnetic flux. ${ }^{10,11}$ Some studies ${ }^{11-17}$ examine repeated measurements of qubit lifetimes under static conditions. However, when discussing these examples, it is important to quantify both the number of counts and the total duration of the measurement. Here, the number of counts relates to the statistical confidence, while the total duration relates to the timescale of fluctuations to which the study is sensitive. Therefore, to confidently report on fluctuations relevant to the calibration period of a quantum processor (for example a few times a day for the IBM Q Experience $\left.{ }^{18}\right)$, we only discuss reports featuring both a large number of counts $(N>1000)$ and a total duration exceeding $5 \mathrm{~h}$.

The first study to satisfy these requirements for relaxation measurements was that of Müller et al., ${ }^{14}$ which revealed that unstable near-resonant two-level-systems (TLS) can induce fluctuations in qubit $T_{1}$. They proposed a model in which the TLS produces a strongly peaked Lorentzian noise profile at the TLS frequency (which is near the qubit frequency). Under the separate model of interacting TLS, ${ }^{19,20}$ the frequency of this near-resonant TLS varies in time. Consequently, the qubit probes the different parts of the TLS-based Lorentzian noise profile, leading to variations in the qubit's $T_{1}$. Although the mechanism was clearly demonstrated, this work ${ }^{14}$ was unable to determine properties of the TLS such as switching rates or dwell times of specific TLS frequency positions. Follow-up work by Klimov et al. ${ }^{17}$ used a tuneable qubit to map the trajectories of individual TLS. These findings ${ }^{17}$ supported the interacting-TLS model and Müller's

\footnotetext{
${ }^{1}$ Microtechnology and Nanoscience, Chalmers University of Technology, SE-412 96 Göteborg, Sweden; ${ }^{2}$ Present address: National Physical Laboratory, Hampton road, Teddington TW11 0LW, UK

Correspondence: Jonas Bylander (bylander@chalmers.se)

These authors contributed equally: Jonathan J. Burnett, Andreas Bengtsson
}

Received: 2 February 2019 Accepted: 3 June 2019

Published online: 26 June 2019 
findings, and were able to clearly determine TLS switching rates, as well as reveal additional diffusive motion of the TLS.

We demonstrate that sufficient statistical analysis can reveal the TLS-based Lorentzian noise spectrum and allow for extraction of switching rates. Importantly, this method does not require a tuneable qubit or advanced reset protocols ${ }^{21}$ and is therefore general to any qubit or setup. Furthermore, the lack of tuning results in a more frequency-stable qubit and consequently less dephasing. This enables us to go beyond the studies of Müller et al. and Klimov et al. by studying the qubit's frequency instabilities due to other noise sources, which reveals a $1 / f$ frequency noise that is remarkably similar to interacting-TLSinduced $1 / f$ capacitance noise found in superconducting resonators. $^{20,22}$ This frequency instability produces a limit on pure dephasing which we observe through sequential inter-leaved measurements of qubit relaxation, dephasing, and frequency.

\section{RESULTS}

Our circuit is made of aluminium on silicon and consists of a single-junction Xmon-type transmon qubit ${ }^{23}$ capacitively coupled to a microwave readout resonator (see the Methods section IV A for more details). The shunt capacitor and the absence of magnetic-flux tunability (absence of a SQUID) effectively decouple the qubit frequency from electrical charge and magnetic flux, reducing the sensitivity to these typical $1 / f$ noise sources. ${ }^{24,25}$ Although these qubits lack frequency tunability, they remain suitable for multi-qubit architectures using all-microwave-based two-qubit-gates. ${ }^{26-29}$ The circuit is intentionally kept simple so that the decoherence is dominated by intrinsic mechanisms and not external ones in the experimental setup. Therefore, there are no individual qubit drive lines, nor any qubit-to-qubit couplings. In addition, both the spectral linewidth of the resonator and the resonator-qubit coupling are kept small, such that photon emission into the resonator (Purcell effect) and dephasing induced by residual thermal population of the resonator are minimised. ${ }^{30} \mathrm{~A}$ detailed experimental setup together with all device parameters are found in the Methods and Table 1.

This study involves two qubits on separate chips which we name $A$ and $B$. The main differences are their Josephson and charging energies and that the capacitor of qubit $B$ was trenched to reduce the participation of dielectric loss. ${ }^{31}$

First we assess the stability of the energy-relaxation time $T_{1}$ by consecutive measurements. The transmon is driven from its ground to first-excited state by a calibrated $\pi$ pulse. The qubit state is then read out with a variable delay. The population of the excited state, as a function of the readout delay, is fit to a singleexponential decay to determine $T_{1}$. Figure 1 shows a $65-\mathrm{h}$ measurement of two separate qubits (in separate sample

\begin{tabular}{|lll|}
\hline Table 1. & Summary of device parameters & \\
\hline Parameters & Qubit A & Qubit B \\
\hline$f_{R}$ & $6.035 \mathrm{GHz}$ & $5.540 \mathrm{GHz}$ \\
$f_{01}$ & $4.437 \mathrm{GHz}$ & $3.759 \mathrm{GHz}$ \\
$f_{12}-f_{01}$ & $-0.226 \mathrm{GHz}$ & $-0.278 \mathrm{GHz}$ \\
$E_{J} / h$ & $13.42 \mathrm{GHz}$ & $8.57 \mathrm{GHz}$ \\
$E_{c} / h$ & $0.201 \mathrm{GHz}$ & $0.235 \mathrm{GHz}$ \\
$E_{J} / E_{C}$ & 66.67 & 36.54 \\
$\epsilon / h$ & $-524 \mathrm{~Hz}$ & $-109 \mathrm{kHz}$ \\
\hline
\end{tabular}

Note: $f_{R}$ is the frequency of the readout resonator and $f_{01}$ that of the qubit's 01 transition. $f_{12}-f_{01}$ is the frequency difference between the qubit's 12 transition and 01 transition. $E_{j}$ is the qubit's Josephson energy, $E_{c}$ its charging energy, $h$ is Planck's constant, and $\epsilon$ its charge dispersion enclosures) that are measured simultaneously. The first observation is that the periods of low- $T_{1}$ values are not synchronised between the two qubits, indicating that the dominant mechanism for $T_{1}$ fluctuations is local to each qubit. (The lack of correlation is quantified in Supplementary Fig. 6). In Fig. 1b, we histogram the $T_{1}$ data: this demonstrates that $T_{1}$ can vary by more than a factor 2 for both qubits, similarly to previous studies. ${ }^{14,17}$

To make a fair comparison of the mean $T_{1}$ for two qubits with different frequencies, we can rescale to quality factors $(Q=$ $\left.2 \pi f_{01} T_{1}\right)$. We see that qubit $B\left(Q=1.67 \times 10^{6}\right)$ has a higher quality factor compared with qubit $A\left(Q=1.29 \times 10^{6}\right)$. However, while the quality of qubit $B$ is higher, qubit $B$ has a lower ratio of Josephson to charging energy (see Table 1), resulting in a larger sensitivity to charge noise and parity effects. ${ }^{32}$ Consequently, qubit $B$ exhibits switching between two different transition frequencies, which was not suitable for later dephasing and frequency instability studies. Therefore, most of the paper focuses on qubit $A$.

We continue by measuring $T_{1}$ consecutively for approximately $128 \mathrm{~h}$, and plot the decays in a colour map (Fig. 2a). Here, the colour map makes some features of the data simpler to visualise. Firstly, the fluctuations are comprised of a switching between different $T_{1}$ values, where the switching is instantaneous, but the dwell time at a particular value is typically between $2 \mathrm{~h}$ and $12.5 \mathrm{~h}$. This behaviour (also seen in Fig. 1a) resembles telegraphic noise with switching rates ranging from 20 to $140 \mu \mathrm{Hz}$. Later, we quantify these rates and their reproducibility.

The white box of Fig. $2 a$ and inset Fig. $2 b$ show this switching behaviour occurring within a single iteration. The decay can be fit to two different values of $T_{1}$, one before the switch and one afterwards. This type of decay profile is found in approximately $3 \%$ of the iterations. In all presented $T_{1}$ values (histograms or sequential plots), the lower $T_{1}$ value is used. This is motivated by quantum algorithms being limited by the shortest-lived qubit.

The black box and inset Fig. 2c highlight a decay-profile that is no longer purely exponential, but instead exhibits revivals. Similar revivals have been observed in both phase ${ }^{33}$ and flux ${ }^{34}$ qubits, and were attributed to coherently coupled TLS residing in one of the qubit junctions. From the oscillations we extract a qubit-to-TLS
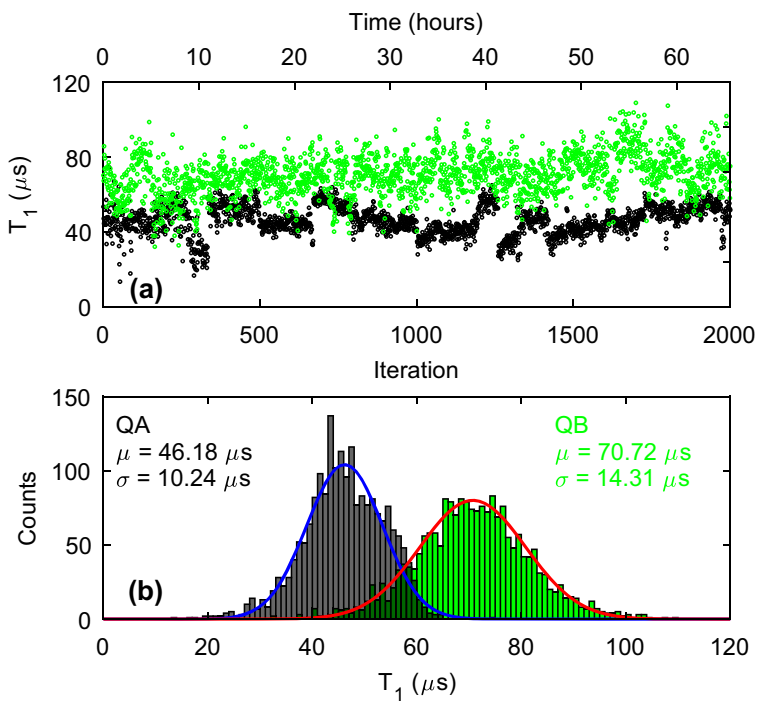

Fig. 1 Synchronous measurement of $T_{1}$ in two separate qubits. a Multiple $T_{1}$ measurements performed simultaneously on qubits $A$ (black) and $\mathrm{B}$ (green). The data consists of 2000 consecutive $T_{1}$ measurements that lasted a total duration of $2.36 \times 10^{5} \mathrm{~s}$ (approximately $65 \mathrm{~h}$ ). $\mathbf{b}$ Histograms of the $T_{1}$ values in $\mathbf{a}$. The histograms have been fit (solid line) to Gaussian distributions with the parameters shown. This data was taken during cooldown 6 


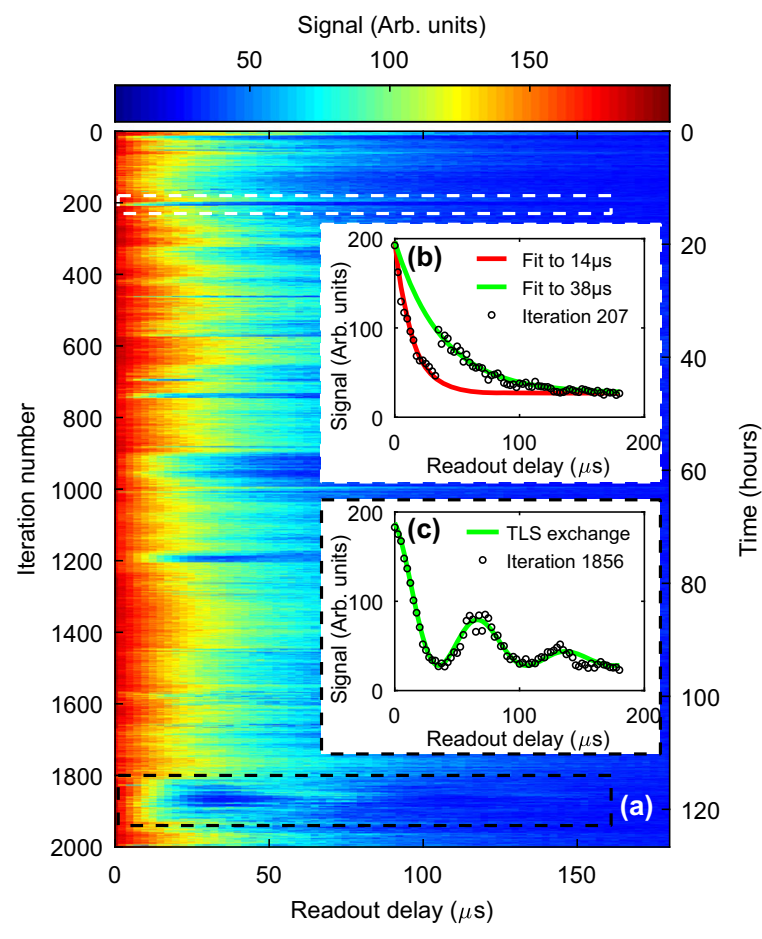

Fig. 2 Raw data of $T_{1}$ decay-profile. a Consecutive $T_{1}$ measurements, spanning $4.6 \times 10^{5} \mathrm{~s}$ (approximately $128 \mathrm{~h}$ ), of qubit A. b A data set showing a change in $T_{1}$ within a single iteration. These jumps are found to occur in approximately $3 \%$ of all measurements. c A data set showing a decaying sinusoidal (rather than a purely exponential) decay profile. The appearance of revivals is due to the resonant exchange with a TLS. These profiles are found to occur in approximately $5 \%$ of all iterations. This data was taken during cooldown 5

coupling of $g_{\mathrm{TLS}}=4.8 \mathrm{kHz}$. Assuming a TLS dipole moment of 1 eA ${ }^{35}$ the coupling corresponds to an electric field line of $39 \mu \mathrm{m}$ (see the Supplementary section C for more details). This length is larger than the Josephson junction; therefore, we conclude that this particular TLS is located on one of the surfaces of the shunting capacitor (not within the junction). Since the invention of transmons and improvement in capacitor dielectrics, individual TLS have only been found to incoherently couple to a transmon, ${ }^{23}$ and the authors are not familiar with any examples of a coherent coupling between a TLS and a transmon.

Approximately $5 \%$ of decay profiles show a clear revival structure, with a further $3 \%$ showing hints of it. Of these, some revival shapes (such as the one shown in the black box) remained stable and persisted for approximately $10 \mathrm{~h}$, whereas others lasted for only 2-3 traces (around $10 \mathrm{~min}$ ). Since the qubit here is fixed in frequency, these appearances/disappearances of the coherent TLS arise due to the TLS shifting in frequency ${ }^{14,17,19,20}$ relative to the static qubit. The observation of coherent oscillations in the decay, and in particular that oscillation periods remained stable for hours (for the same duration as the $T_{1}$ fluctuations), constitutes clear evidence for TLS being the origin of the $T_{1}$ fluctuations, in agreement with both the Müller ${ }^{14}$ and Klimov ${ }^{17}$ results.

To gain further insight into these fluctuations we perform statistical analysis commonly used in the field of frequency metrology. In parallel, we examine both the overlapping Allan deviation (Fig. 3a) and the spectral properties (Fig. 3b) of the $T_{1}$ fluctuations. Allan deviation is a standard tool for identifying different noise sources in e.g. clocks and oscillators. ${ }^{36}$ Here, we introduce the Allan deviation as a tool to identify the cause of fluctuations in qubits. The most striking feature in Fig. $3 a$ is the peak and subsequent decay around $\tau=10^{4} \mathrm{~s}$. Importantly, no
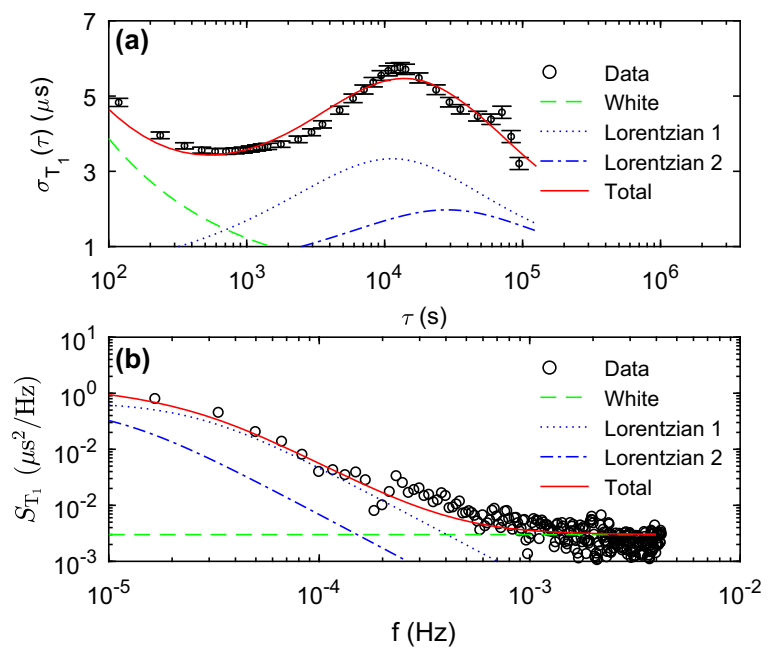

Fig. 3 Time and frequency domain analysis of $T_{1}$ fluctuation. Statistical analysis of 2001 sequential $T_{1}$ measurements of qubit $A$ spanning a total measurement duration of $2.36 \times 10^{5} \mathrm{~s}$. a Overlapping Allan deviation of $T_{1}$ fluctuations. b Welch-method spectral density of $T_{1}$ fluctuations. In both plots there are fits to the total noise (red line) which is formed of white noise (green lines) and two different Lorentzians (blue lines). The amplitudes and time constants of all noise processes are the same for both types of analysis. The error-bars of the Allan deviation correspond to 1 standard deviation. This data was taken during cooldown 2

Table 2. Summary of the noise parameters for modelling $T_{1}$ fluctuations

\begin{tabular}{|c|c|c|c|c|c|}
\hline Data & $h_{0}\left(\mu s^{2} / \mathrm{Hz}\right)$ & $1 / \tau_{0}^{\operatorname{Lor} 1}(\mu \mathrm{Hz})$ & $A^{\text {Lor1 }}(\mu \mathrm{s})$ & $1 / \tau_{0}^{\text {Lor2 }}(\mu \mathrm{Hz})$ & $A^{\operatorname{Lor2}(\mu \mathrm{s})}$ \\
\hline QA_C2 & $3.0 \times 10^{-3}$ & 158.7 & 5.4 & 80.6 & 3.2 \\
\hline QA_C3 & $2.6 \times 10^{-3}$ & 200.0 & 2.4 & 100.0 & 4.5 \\
\hline QA_C5 & $2.0 \times 10^{-3}$ & 142.9 & 5.2 & 83.3 & 2.6 \\
\hline QA_C6 & $1.2 \times 10^{-3}$ & 333.3 & 4.5 & 71.4 & 1.8 \\
\hline QB_C1 & $1.3 \times 10^{-2}$ & 1851.8 & 2.5 & - & - \\
\hline QB_C5 & $1.4 \times 10^{-2}$ & 1000.0 & 3.2 & 90.9 & 6.6 \\
\hline QB_C6 & $5.7 \times 10^{-3}$ & 1111.1 & 4.2 & 76.9 & 2.2 \\
\hline
\end{tabular}

power-law noise process can produce such a peak; instead, it is an unambiguous sign of a Lorentzian noise process. Such Lorentzianlike switching was observed in the $T_{1}$-vs.-time measurement in Fig. 1 a. In Fig. 3, we model the noise with two Lorentzians with a white noise floor, and apply the modelled noise to both the spectrum and the Allan deviation. Therefore, the noise parameters are the same for both plots: the Methods section has more details on the scaling of Lorentzian noise between the Allan and spectral analysis methods. From Fig. 3, we obtain Lorentzian switching rates of 80.6 and $158.7 \mu \mathrm{Hz}$.

Within Fig. 4 and Table 2, we show the reproducibility of these features across thermal cycles. Collectively, we find switching rates ranging from $71.4 \mu \mathrm{Hz}$ to $1.9 \mathrm{mHz}$-slower than those obtained by measurements of charge noise ${ }^{37}$ but similar to bulk-TLS dynamics ${ }^{38,39}$ and in agreement with rates determined from measurements tracking the time evolution of individual TLS. ${ }^{17}$ These measurements demonstrate not only that superconducting 

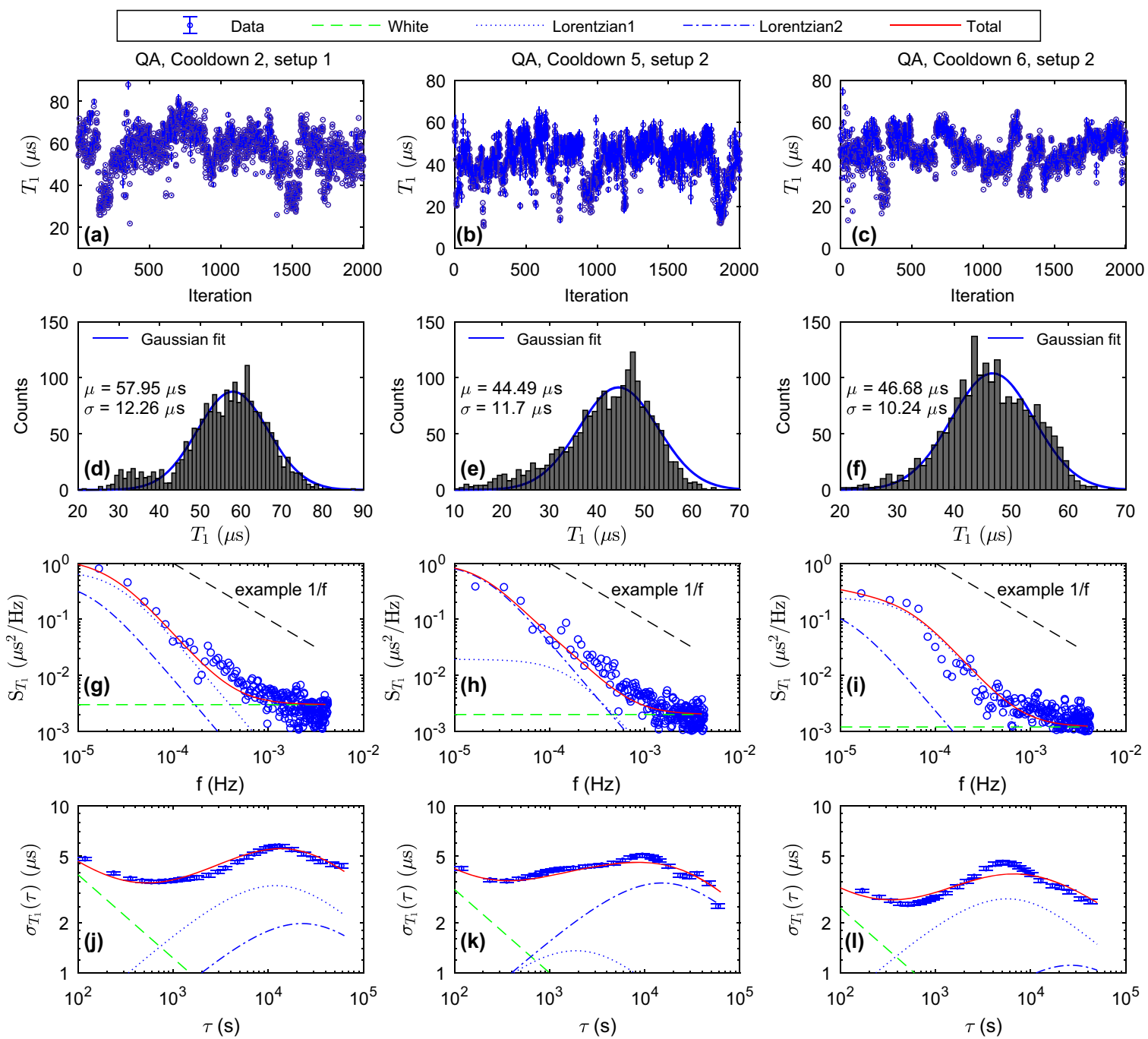

Fig. 4 Reproducibility of $T_{1}$ fluctuations in qubit A across separate cooldowns. a-c Time evolution of $T_{1}$ vs. iteration. d-f Statistics of $T_{1}$ plotted as a histogram, with a Gaussian fit. $\mathbf{g}$-i Welch spectral density estimate of the $T_{1}$ fluctuations. $\mathbf{j}-\mathbf{I}$ Overlapping Allan deviation of $T_{1}$ fluctuations. Across $\mathbf{g}-\mathbf{i}$ and $\mathbf{j}-\mathbf{I}$ the noise model is the same, where the parameters can be found within Table 2 . For illustrative purposes, we include a $1 / f$ noise guideline within $\mathbf{g}-\mathbf{i}$. The error-bars of the time evolution of $T_{1}$ vs. iteration correspond to 1 standard deviation. Similar data for qubit B can be found within Supplementary Fig. 2

qubits are useful probes of TLS, but unambiguously demonstrate the role of a TLS-based Lorentzian noise profile as a limiting factor to the temporal stability of qubit coherence.

In addition to studying $T_{1}$ fluctuations, we also explore fluctuations in qubit frequency and dephasing. To this end, we measure the qubit frequency and the characteristic decay time $T_{2}^{*}$ by means of a de-tuned Ramsey fringe. We interleave the Ramsey sequence, point-by-point, with the previously discussed $T_{1}$ relaxation sequence. For clarity, if we consider the energyrelaxation measurements in Fig. 2, the main plot (a) represents the complete measurement set, which is formed from 2000 iterations. Each iteration (e.g., either inset) consists of data points which are themselves the averaged results of 1000 repeated measurements. In the interleaved sequence, we measure the data point in the $T_{1}$ sequence and then the data point in the Ramsey sequence for each delay time (i.e., the time between the $\pi$ pulse and readout, in the $T_{1}$ measurement, and in-between the $\pi / 2$ pulses, in the Ramsey $T_{2}^{*}$ measurement). This sequence is then looped through all values of the delay time to map out the $T_{1}$ and Ramsey decay profiles (i.e., the iteration). While averaging each point in the inner loop gives a longer iteration time, and increases the noise window to which the Ramsey fringe is sensitive, ${ }^{40}$ it allows for all qubit parameters to be known in each iteration. From the so-obtained $T_{1}$ and $T_{2}^{*}$ we calculate the pure-dephasing time $T_{\phi}$ from $1 / T_{2}^{*}=1 / 2 T_{1}+1 / T_{\phi}$. These values are shown in Fig. $5 \mathrm{~b}$, and the histogram of $T_{2}^{*}$ values is shown in Fig. 5c.

In Fig. 5a we have extracted, from the Ramsey fringes, the frequency motion of the qubit relative to its mean frequency $\left(f_{01}-\overline{f_{01}}\right)$. In general, the observed frequency shifts are on the order of 1 to $3 \mathrm{kHz}$, with infrequent shifts of up to $20 \mathrm{kHz}$. A histogram of the qubit frequency (Fig. $5 \mathrm{~d}$ ) reveals a main peak with a full-width at half-maximum of approximately $2 \mathrm{kHz}$. These frequency shifts are significantly smaller than the approximately $500 \mathrm{kHz}$ frequency instability found in flux-tuneable qubits. ${ }^{17}$ From the perspective of gate fidelity, a $1-\mathrm{kHz}$ frequency shift should have negligible effect, meaning that our qubits are well suited for quantum information processing since no re-calibration of the qubit frequency is needed. However, a fluctuating qubit frequency necessarily leads to qubit dephasing so it is important to quantify this fluctuation and therefore aid in efforts to find, and mitigate, the noise source.

To provide more information on possible mechanisms for the frequency instability, we examine both the overlapping Allan deviation (Fig. 5e) and the spectrum of frequency fluctuations 

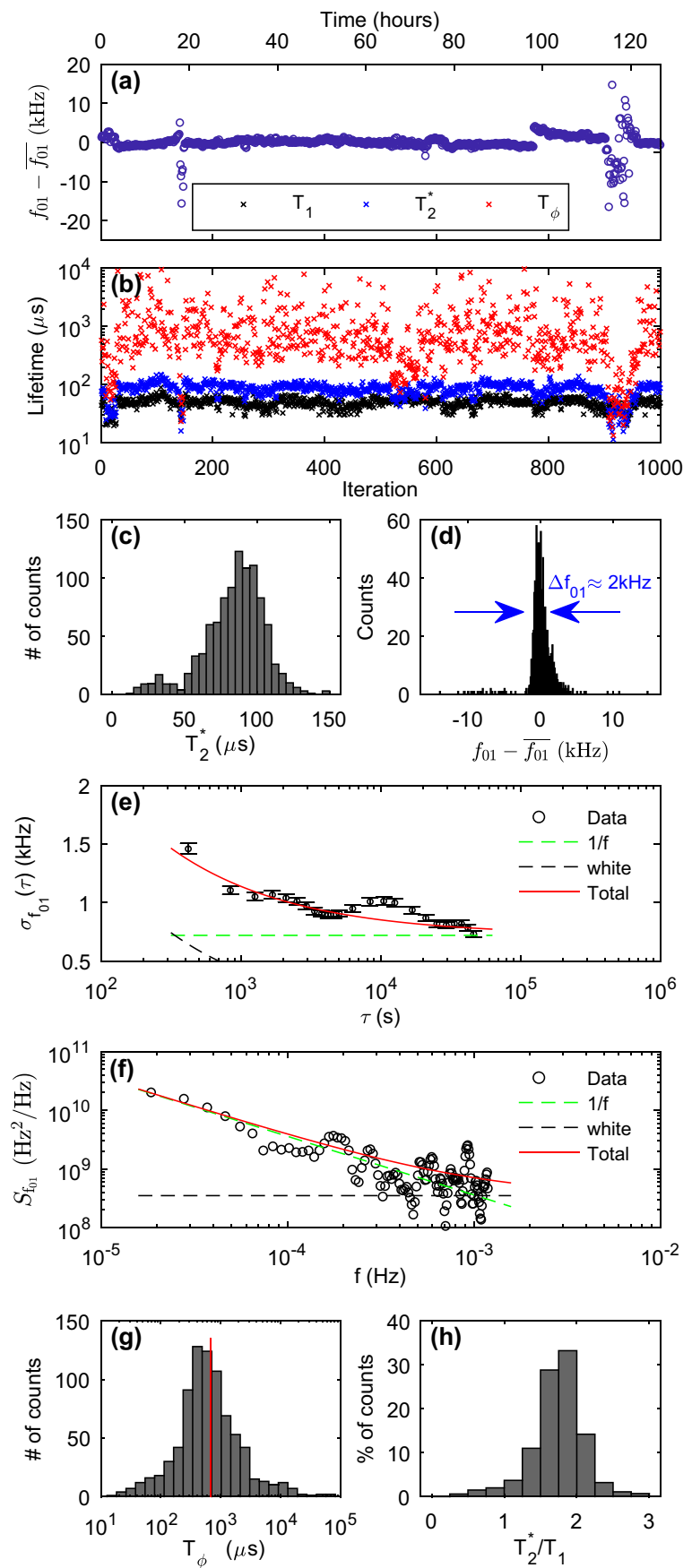

Fig. 5 An interleaved series of $1000 T_{1}$ relaxation and $T_{2}^{*}$ Ramsey measurements of qubit A. a Qubit frequency $\left(f_{01}\right)$ shift relative to its mean $\left(\overline{f_{01}}\right)$ determined from the Ramsey experiments. b Extracted $T_{1}$ (black), $T_{2}^{*}$ (blue), and $T_{\phi}$ (red). c Histogram of $T_{2}^{*}$ from the data in $\mathbf{b}$. d Histogram of the data in $\mathbf{a}$. The frequency fluctuations from a are analysed by overlapping Allan deviation (e) and by Welch-method spectrum (f). The solid and dashed lines represent the modelled noise, where the noise amplitudes are the same for both types of analysis. $\mathbf{g}$ Histogram of $T_{\phi}$ from the data in $\mathbf{b}$. The solid line indicates the $T_{\phi}$ limit calculated by integrating the frequency noise from e. h Histogram of $T_{2}^{*} / T_{1}$ from the data in $\mathbf{b}$. We find $1.4<T_{2}^{*} / T_{1}<2.2$ in $81.7 \%$ of the counts. This data was taken during cooldown 3

(Fig. 5f). In red, the frequency noise is modelled to $A / f+B$, where the exponent of $f$ is 1 . Similarly to the previous $T_{1}$ analysis, the noise model is scaled so that the red line has the same amplitude in both Fig. 5e, f. In this model, the $1 / f$ noise amplitude is $A=3.6 \times 10^{5} \mathrm{~Hz}^{2}$.

\section{DISCUSSION}

For both qubits, across all cooldowns, we found fluctuations in $T_{1}$ that could be described by Lorentzian noise with switching rates in the range from $75 \mu \mathrm{Hz}$ to $1 \mathrm{mHz}$. For all superconducting qubits, three relaxation channels are usually discussed: TLS, quasiparticles, and parasitic microwave modes. Of these, parasitic microwave modes should not cause fluctuation since they are defined by the physical geometry. For quasiparticles in aluminium, we can compare our observed slow fluctuations with quasiparticle mechanisms found in the qubit literature: the quasiparticle recombination rate is $1 \mathrm{kHz} ;{ }^{41}$ the timescale of quasiparticle number fluctuations leads to rates in the range from $0.1 \mathrm{kHz}$ to $10 \mathrm{kHz}$; and finally, quasiparticle tunnelling (parity switching events) in transmons have rates in the range $0.1 \mathrm{kHz}$ to $30 \mathrm{kHz} .^{32}$ Therefore, fluctuations in the properties of the superconductor occur over rates which differ by over six orders of magnitude compared to those found in our experiment. Instead, we highlight that, at low temperatures, bulk-TLS dynamics ${ }^{38,39}$ and TLS-charge noise ${ }^{34,37}$ vary over long timescales equivalent to rates in the range from $10 \mathrm{mHz}$ to $100 \mathrm{~Hz}$.

The observed coherent qubit-TLS coupling (Fig. 2c) is an unambiguous sign of the existence of near-resonant TLS. Its fluctuation follows similar time constants as the $T_{1}$ fluctuations, which constitutes clear evidence of spectral instability, as expected from the interacting-TLS model. ${ }^{19,20}$ We therefore attribute the origin of the $T_{1}$ decay to near-resonant TLS, and the Lorentzian fluctuations in the qubit's $T_{1}$ (shown in Figs. 3 and $4 \mathrm{~g}-\mathrm{l})$ arise due to spectral instabilities of the TLS as described by Müller et al. ${ }^{14}$ The extracted switching rates then represent the rate at which the near-resonant TLS is changing frequency. Similarly, the quality factor of superconducting resonators has also been found to vary ${ }^{42}$ due to spectrally unstable TLS.

In general, we find that two separate Lorentzians are required to describe the fluctuation. This does not necessarily imply the existence of two near-resonant TLS-instead it is a limitation of the analysis, as we cannot resolve the difference between, say, two near-resonant TLS, each with two preferential frequencies, vs. one near-resonant TLS that has four preferential frequencies. Such a difference could be inferred by measuring the local density of near-resonant TLS, ${ }^{17}$ although such a measurement has demonstrated that both scenarios above are possible. ${ }^{17}$ In addition, when repeating the measurements across multiple cooldowns, we consistently find a near-resonant TLS that follows similar switching statistics. Between each cooldown, the TLS configuration is expected to completely change. Essentially, this means that the detuning and coupling of the observed near-resonant TLS should vary for each cooldown. However, despite any expected reconfiguration, at least one spectrally unstable near-resonant TLS is always found to exist.

When examining the frequency stability of qubit A, we found a frequency noise of approximately $2 \mathrm{kHz}$, which was well described by a $1 / f$ amplitude of $3.6 \times 10^{5} \mathrm{~Hz}^{2}$ (Fig. $5 \mathrm{~d}-\mathrm{f}$ ). Typically, dephasing is thought to arise due to excess photons within the cavity, ${ }^{9,43}$ flux noise, ${ }^{25}$ charge noise, ${ }^{34,37}$ quasiparticles tunnelling through the Josephson junctions, ${ }^{32}$ or the presence of excess quasiparticles. ${ }^{44}$ For qubit $A$, the charge dispersion is calculated to $524 \mathrm{~Hz}$, much smaller than most of the observed frequency shifts. This rules out charge noise and tunnelling quasiparticles as the main source of the observed frequency fluctuations. Quasiparticle fluctuations have been extensively studied, ${ }^{41}$ where the magnitude of frequency shifts scales with the kinetic inductance. Therefore, while they can be of order $100 \mathrm{kHz}$ in disordered superconductors, ${ }^{45}$ they are much smaller in elemental superconductors. In fact, recent experiments ${ }^{41}$ showed that the quasiparticles in aluminium produced an un-measurably small frequency shift; instead, the quasiparticles' influence was revealed only by examining correlated amplitude and frequency noise. Therefore, not only do quasiparticles produce immeasurably small frequency 
shifts, but, as noted earlier, they act over much shorter timescales (i.e., rates are equivalent to $\mathrm{kHz}^{32,41}$ rather than the sub- $\mathrm{mHz}$ timescales observed here).

Instead we highlight two further TLS-based mechanisms. Firstly, TLS within the Josephson junction can cause critical-current noise ${ }^{46}$ which can produce a frequency noise by modulating the Josephson energy. Alternatively, superconducting resonators demonstrate that TLS can produce frequency instabilities ${ }^{20,22}$ (capacitance noise). Both of these mechanisms exhibit a $1 / f$ noise, where the noise amplitude is close to that which we find here. One could distinguish between these two effects by examining the temperature dependence of the qubit's frequency noise. Here, critical current noise ${ }^{46}$ scales $\propto T$ while capacitance noise ${ }^{20,22}$ scales $\propto 1 / T^{1.3}$.

Irrespective of the origin of the frequency instability, the noise spectrum in Fig. $5 f$ can be integrated to estimate the pure dephasing of the qubit. ${ }^{47}$ From this calculation, the expected $T_{\phi}$ is $0.8 \mathrm{~ms}$. In Fig. $5 \mathrm{~g}$ we histogram the $T_{\phi}$ to reveal a peak around $0.7 \mathrm{~ms}$, with diminishing counts above $1 \mathrm{~ms}$, in good agreement with the estimate from the integrated frequency noise.

In Fig. 5b, c, $T_{2}^{*}$ is almost always longer than $T_{1}$, implying that $T_{\phi}$ $>2 T_{1}$. In Fig. $5 \mathrm{~h}$ this is quantified, as the histogram of the ratio of $T_{2}^{*} / T_{1}$ reveals that the qubit dephasing is almost always near $2 T_{1}$. Therefore, the qubit's $T_{2}^{*}$ is mainly limited by $T_{1}$. To the authors' knowledge all other demonstrations of $T_{1}$-limited $T_{2}$ required dynamical decoupling by either a Hahn-echo (spin-echo) ${ }^{9,25}$ or $\mathrm{CPMG}^{11}$ sequence. However, neither of those works provide any statistics on whether the qubits were always $T_{1}$-limited. The histogram in Fig. $5 \mathrm{~h}$ also reveals counts where the ratio is above 2: these correspond to the instances where the $T_{1}$ has fluctuated within an iteration, similar to that shown in Fig. $2 \mathrm{~b}$.

In summary, we have measured the stability of qubit lifetimes across more cooldowns and for measurement spans longer than previous studies. Collectively, this demonstrates that qubit fluctuations, due to spectrally unstable TLS, are consistently observed, even when $T_{1}$ is high (approaching $100 \mu \mathrm{s}$ ). The spectral motion of TLS is particularly problematic for gates requiring frequency movement (e.g., $\mathrm{CPHASE}^{3}$ ). Here, leakage to TLS during gate operations, and the optimum idling frequencies become time-varying on a per-qubit basis. In addition, since gate fidelities contain a non-negligible error contribution from $T_{1}{ }_{1}^{48}$ the optimal gate duration will be affected by $T_{1}$ varying in time. Therefore, these fluctuations demonstrate why it is necessary to re-calibrate qubits every few hours. Fundamentally, this also demonstrates that future reports on qubit coherence times require not only statistics for reproducibility, but also that the measurement duration should exceed several hours in order to adequately report the typical rather than exceptional coherence time.

Note added-Recently, a preprint on comparable observations was published by Schlör et al., ${ }^{49}$ who independently demonstrated that single fluctuators (TLS) are responsible for frequency and dephasing fluctuations in superconducting qubits. In addition, another recent preprint by Hong et al., ${ }^{50}$ specifically measures fluctuations in gate fidelity and independently identifies $T_{1}$ fluctuation of the underlying qubits as the probable cause.

\section{METHODS}

\section{Experimental details}

The qubits are fabricated out of electron-beam evaporated aluminium on a high-resistivity intrinsic silicon substrate. Everything except the Josephson junction is defined using direct-write laser lithography and etched using wet chemistry. The Josephson junction is defined in a bi-layer resist stack using electron-beam lithography, and later deposited using a two-angle evaporation technique that does not create any extra junctions or floating islands. ${ }^{51}$ An additional lithography step is included to ensure a superconducting contact between the junction and the rest of the circuit; after the lithography, but prior to deposition of aluminium, an argon ion

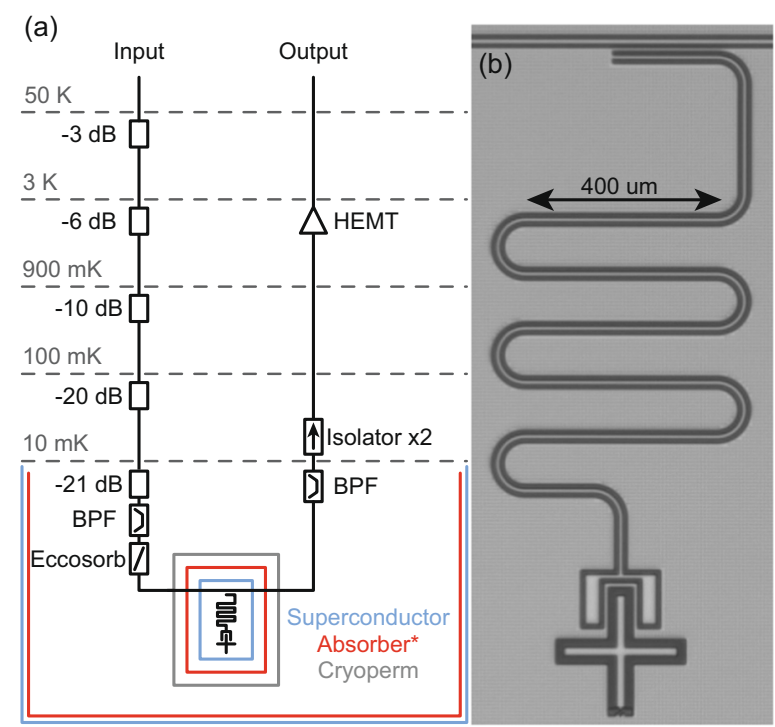

Fig. 6 An overview of the experiment. a Simplified schematic of the experimental setup. The main features are the various shielding layers. The absorber coating (red and with an asterisk) was added for setup 2. b Optical image of the qubit sample. It shows a common microwave transmission line, a $\lambda / 4$ resonator and a transmon qubit with a coplanar capacitor (Xmon-geometry). The arrow represents a 400 micron scale bar

mill is used to remove native aluminium oxide. This avoids milling underneath the junction, which has been shown to increase the density of TLS. $^{10}$ Finally, the wafer is diced into individual chips and cleaned thoroughly using both wet and dry chemistry. Moreover, qubit B underwent a trenching step where approximately $1 \mu \mathrm{m}$ of the silicon dielectric was removed from both the qubit and the resonator using an fluorine based reactive-ion etch. ${ }^{52}$

A simplified schematic of the experimental setup is shown in Fig. 6a. The samples sit within a superconducting enclosure, which itself is inside of an absorber-lined radiation shield and a cryoperm layer. This is located within a further absorber-lined radiation shield and a further superconducting layer which encloses the entire mixing chamber. Everything inside the cryoperm layer (screws, sample enclosures, and cables) is non-magnetic. The setup, including absorber recipe, is similar to a typical qubit box-in-abox setup. ${ }^{44}$ For the different cooldowns, two setups (labelled 1 and 2 ) were used. Setup 2 was as described above, whereas setup 1 lacked the absorber coating marked with a red asterisk in Fig. 6 a.

\section{Data handling}

The qubit decoherence data is processed in the following way. First, the digitiser signal is rotated to one quadrature. Next, the signal is normalised to the maximum visibility of the qubit $|0\rangle$ and $|1\rangle$ states. Then, for qubit relaxation data, a fit to a single exponential is performed. Within Fig. $4 a-c$, $T_{1}$ data is presented with error-bars. These error-bars correspond to 1 standard deviation, determined from confidence intervals of the exponential fit. For the Ramsey measurements, the initial processing is as described above. However, the Ramsey frequency $\left(f_{\text {Ram }}\right)$ is initially determined by FFT of the data. The resulting frequency from the FFT is used as an initial frequency guess to a model of the form:

$$
\left\langle P_{e}(t)\right\rangle=\exp \left(-t / T_{2}^{*}\right) \cos \left(2 \pi f_{\text {Ram }}+\phi_{0}\right)
$$

where $\phi_{0}$ is a phase offset that is generally zero. Across all of the data-sets examined for qubit $A$, the FFT reveals only one oscillation frequency, whereas for qubit $B$, two frequencies are observed due to a larger charge dispersion. Consequently, Eq. (1) does not fit well for qubit B, and we omit qubit $B$ from the dephasing and frequency results.

For the qubit relaxation, we did attempt fits to a double exponential model. ${ }^{11,53}$ Within this model, an additional relaxation channel due to quasiparticles near the junction can lead to a skewing of the decay-profile. Here, we found the confidence interval for numerous parameters was unphysically large, indicating that the model over-parameterised our data. Therefore, we continued to use the single-exponential model. However, 
this is not surprising as the double-exponential is typically used for fluxqubits and fluxoniums, rather than the single-junction transmon-type qubit studied here.

\section{Sample handling}

Here, we clarify the sample handling across the entire experiment. For each qubit sample, after completing fabrication, they were covered in protective resist until the morning of their first cooldown (cooldown 1 for qubit $B$ and cooldown 2 for qubit A). After removal of the resist, the samples were wirebonded within a sample enclosure. Once sealed, the samples remained within their enclosures and were kept attached to the fridge for the entire experimental run. Therefore, when the fridge was warm, the samples were kept at the ambient conditions of the lab. Qubit B was not measured between cooldown 2 and cooldown 5 , although it was still cooled down. However, qubit B was examined again in cooldowns 5 and 6 to gather statistics on the reproducibility of parameter fluctuations.

\section{Spectral and Allan analysis}

Within the main text, information on TLS switching rates is inferred by examining the reproducibility of coherence parameters. Primarily, this is obtained by examining the Allan statistics and spectral properties of $T_{1}$ fluctuations. Here, the same data set is used to produce a plot of a Welchmethod FFT $\left(S_{T_{1}}(f)\right)$ and an overlapping Allan deviation $\left(\sigma_{T_{1}}(\tau)\right)$. For the Welch analysis, the quantity analysed is $T_{1}-\overline{T_{1}}$ (or for frequency it is $\left.f_{01}-\overline{f_{01}}\right)$. Therefore, the analysed quantity is not presented in fractional units. Consequently, the units of spectral analysis are $\mu \mathrm{s}^{2} / \mathrm{Hz}$ for $T_{1}$ fluctuations $\left(\mathrm{Hz}^{2} / \mathrm{Hz}\right.$ for frequency analysis). Equivalently, the Allan statistics are presented in units of $\mu$ s for $T_{1}$ fluctuation $(\mathrm{Hz}$ for frequency fluctuation).

The Allan deviation offers a few advantages compared with the spectrum. The method is directly traceable in that the Allan methods use simple mathematical functions that do not require any careful handling of window functions or overlap. When examining low-frequency processes, this eases a considerable burden in FFT-analysis which is to distinguish real features from remnants of window functions. This traceability is core to the usage within the frequency metrology community. The Allan method also provides clear error bars (defined as equal to 1 standard deviation), which translate to an efficient use of the data with optimum averaging of all data that shares a common separation, that is, all data pairs for any separation ( $\tau$ in the Allan plot) are averaged over. Moreover, the Allan method can distinguish linear drift from any other divergent noise processes. Within an FFT, a linear drift appears as a general $1 / f^{a}$ slope where $a$ is not unique compared with other noise sources. Within the Allan, a linear drift appears as $\tau^{a}$ where $a$ is distinct and unique compared with other divergent noise types.

From here, beginning with the Allan deviation, we consider the standard power-law model ${ }^{36}$ of noise processes,

$$
\begin{gathered}
\sigma_{T_{1}}(\tau)=\left(\frac{h_{0}}{2}\right)^{\frac{1}{2}} \tau^{-\frac{1}{2}} \\
+\left(2 \ln (2) h_{-1}\right)^{\frac{1}{2}} \\
+\left(\frac{(2 \pi)^{2}}{6} h_{-2}\right)^{\frac{1}{2}} \tau^{\frac{1}{2}}
\end{gathered}
$$

which can also be represented as spectral noise

$$
S_{T_{1}}(f)=\frac{h_{-2}}{f^{2}}+\frac{h_{-1}}{f}+h_{0}
$$

where, in frequency metrology notation, $h_{-2}$ is the amplitude of a random walk noise process, $h_{-1}$ is the amplitude of a $1 / f$ noise process and $h_{0}$ is the amplitude of white noise.

In general terms, the power-law noise processes create a well-like shape in the Allan analysis, where, with the terms listed above, the walls have slopes of $\propto \tau^{-\frac{1}{2}}$ and $\propto T^{\frac{1}{2}}$. If more terms are included in the power-law noise model, the available slope gradients increase, but the well-like shape remains. When applied to the $T_{1}$ fluctuations (Fig. 3), this model is not able to describe the most striking feature: the hill-like peak with subsequent second decreasing slope. Within Allan analysis, the rise and fall of a single peak can only be represented by a Lorentzian noise process. Therefore, starting from

$S(f)=\frac{4 A^{2} \tau_{0}}{1+\left(2 \pi f \tau_{0}\right)^{2}}$

where $A$ represents the Lorentzian noise amplitude and $\tau_{0}$ is the characteristic timescale, Lorentzian noise can be represented in Allan deviation by ref. ${ }^{54}$.

$\sigma(\tau)=\frac{A \tau_{0}}{\tau}\left(4 e^{\left(-\tau / \tau_{0}\right)}-e^{\left(-2 \tau / \tau_{0}\right)}-3+2 \tau / \tau_{0}\right)^{1 / 2}$

From here, we model the $T_{1}$ fluctuations by two separate Lorentzians and white noise. When plotted, the noise from these sources is identical (i.e., the same $h_{0}, A$, and $\tau_{0}$ ) for both the Welch-FFT and Allan deviation. For the rest of the data sets, we tabulate the Lorentzian parameters and white noise level in Table 2.

\section{DATA AVAILABILITY}

The data that supports the findings of this study is available from the corresponding authors upon reasonable request.

\section{CODE AVAILABILITY}

The code that supports the findings of this study is available from the corresponding authors upon reasonable request.

\section{ACKNOWLEDGEMENTS}

We wish to express our gratitude to Philip Krantz and Tobias Lindström for insightful discussions. We acknowledge financial support from the Knut and Alice Wallenberg Foundation, the Swedish Research Council, and the EU Flagship on Quantum Technology H2020-FETFLAG-2018-03 project 820363 OpenSuperQ. In addition, J.J.B acknowledges financial support from the Industrial Strategy Challenge Fund Metrology Fellowship as part of the UK government's Department for Business, Energy and Industrial Strategy.

\section{AUTHOR CONTRIBUTIONS}

J.J.B. and A.B. are considered co-first authors. J.J.B., A.B., and J.B. planned the experiment, A.B. designed the samples, A.B. fabricated the samples with input from J.J.B. The experiments were mainly performed by J.J.B. and A.B. with help from M.S., D.N., and M.K. Analysis was performed by J.J.B. and A.B. with input from P.D. and J.B. The manuscript was written by J.J.B., A.B., and J.B. with input from all authors. J.B. and P.D. provided support for the work.

\section{ADDITIONAL INFORMATION}

Supplementary information accompanies the paper on the npj Quantum Information website (https://doi.org/10.1038/s41534-019-0168-5).

Competing interests: The authors declare no competing interests.

Publisher's note: Springer Nature remains neutral with regard to jurisdictional claims in published maps and institutional affiliations.

\section{REFERENCES}

1. Preskill, J. Reliable quantum computers. Proc. R. Soc. London Ser A 454, 385-410 (1998).

2. Fowler, A. G., Mariantoni, M., Martinis, J. M. \& Cleland, A. N. Surface codes: towards practical large-scale quantum computation. Phys. Rev. A 86, 032324 (2012).

3. Barends, R. et al. Superconducting quantum circuits at the surface code threshold for fault tolerance. Nature 508, 500-503 (2014).

4. Rol, M. A. et al. Restless tuneup of high-fidelity qubit gates. Phys. Rev. Appl. 7, 041001 (2017).

5. Preskill, J. Quantum computing in the NISQ era and beyond. Quantum 2, 79 (2018).

6. Martinis, J. M. Qubit metrology for building a fault-tolerant quantum computer NPJ Quantum Inf. 1, 15005 (2015).

7. Oliver, W. D. \& Welander, P. B. Materials in superconducting quantum bits. MRS Bull. 38, 816-825 (2013).

8. Gu, X., Kockum, A. F., Miranowicz, A., Liu, Y.-x \& Nori, F. Microwave photonics with superconducting quantum circuits. Phys. Rep. 718, 1-102 (2017).

9. Wang, Z. et al. Cavity attenuators for superconducting qubits. Phys. Rev. Appl. 11 014031 (2019).

10. Dunsworth, A. et al. A method for building low loss multi-layer wiring for superconducting microwave devices. Appl. Phys. Lett. 112, 063502 (2018). 
11. Yan, F. et al. The flux qubit revisited to enhance coherence and reproducibility. Nat. Commun. 7, 12964 (2016).

12. Dial, O. et al. Bulk and surface loss in superconducting transmon qubits. Supercond. Sci. Technol. 29, 044001 (2016).

13. Rosenberg, D. et al. 3D integrated superconducting qubits. NPJ Quantum Inf. 3, 42 (2017).

14. Müller, C., Lisenfeld, J., Shnirman, A. \& Poletto, S. Interacting two-level defects as sources of fluctuating high-frequency noise in superconducting circuits. Phys. Rev. B 92, 035442 (2015).

15. Gustavsson, S. et al. Suppressing relaxation in superconducting qubits by quasiparticle pumping. Science 354, 1573-1577 (2016).

16. Chang, J. B. et al. Improved superconducting qubit coherence using titanium nitride. Appl. Phys. Lett. 103, 012602 (2013).

17. Klimov, P. V. et al. Fluctuations of energy-relaxation times in superconducting qubits. Phys. Rev. Lett. 121, 090502 (2018).

18. IBM Q. IBM Q Experience website. https://www.research.ibm.com/ibm-q/. Accessed July 2018.

19. Faoro, L. \& loffe, L. B. Interacting tunneling model for two-level systems in amorphous materials and its predictions for their dephasing and noise in superconducting microresonators. Phys. Rev. B 91, 014201 (2015).

20. Burnett, J. et al. Evidence for interacting two-level systems from the $1 / \mathrm{f}$ noise of a superconducting resonator. Nat. Commun. 5, 4119 (2014).

21. Geerlings, K. et al. Demonstrating a driven reset protocol for a superconducting qubit. Phys. Rev. Lett. 110, 120501 (2013).

22. de Graaf, S. E. et al. Suppression of low-frequency charge noise in superconducting resonators by surface spin desorption. Nat. Commun. 9, 1143 (2018).

23. Barends, R. et al. Coherent Josephson qubit suitable for scalable quantum integrated circuits. Phys. Rev. Lett. 111, 080502 (2013).

24. Gustafsson, M. V., Pourkabirian, A., Johansson, G., Clarke, J. \& Delsing, P. Thermal properties of charge noise sources. Phys. Rev. B 88, 245410 (2013).

25. Bylander, J. et al. Noise spectroscopy through dynamical decoupling with a superconducting flux qubit. Nat. Phys. 7, 565-570 (2011).

26. McKay, D. C. et al. Universal gate for fixed-frequency qubits via a tunable bus. Phys. Rev. Appl. 6, 064007 (2016).

27. Chow, J. M. et al. Simple all-microwave entangling gate for fixed-frequency superconducting qubits. Phys. Rev. Lett. 107, 080502 (2011).

28. Economou, S. E. \& Barnes, E. Analytical approach to swift nonleaky entangling gates in superconducting qubits. Phys. Rev. B 91, 161405(R) (2015).

29. Paik, $\mathrm{H}$. et al. Experimental demonstration of a resonator-induced phase gate in a multiqubit circuit-QED system. Phys. Rev. Lett. 117, 250502 (2016).

30. Clerk, A. A. \& Utami, D. W. Using a qubit to measure photon-number statistics of a driven thermal oscillator. Phys. Rev. A 75, 042302 (2007).

31. Barends, R. et al. Minimal resonator loss for circuit quantum electrodynamics. Appl. Phys. Lett. 97, 023508 (2010).

32. Riste, D. et al. Millisecond charge-parity fluctuations and induced decoherence in a superconducting transmon qubit. Nat. Commun. 4, 1913 (2013).

33. Cooper, K. et al. Observation of quantum oscillations between a Josephson phase qubit and a microscopic resonator using fast readout. Phys. Rev. Lett. 93, 180401 (2004).

34. Gustavsson, S. et al. Dynamical decoupling and dephasing in interacting twolevel systems. Phys. Rev. Lett. 109, 010502 (2012).

35. Martinis, J. et al. Decoherence in Josephson qubits from dielectric loss. Phys. Rev. Lett. 95, 210503 (2005).

36. Rubiola, E. Phase noise and frequency stability in oscillators. In The Cambridge RF and Microwave Engineering Series (Cambridge University Press, 2009). https://www. cambridge.org/core/books/phase-noise-and-frequency-stability-inoscillators/445C 12C4ECBFCD7765116E61561ECOFE https://doi.org/10.1017/CBO9780511812798.
37. Kafanov, S., Brenning, H., Duty, T. \& Delsing, P. Charge noise in single-electron transistors and charge qubits may be caused by metallic grains. Phys. Rev. B 78, 125411 (2008).

38. Salvino, D. J., Rogge, S., Tigner, B. \& Osheroff, D. D. Low temperature ac dielectric response of glasses to high dc electric fields. Phys. Rev. Lett. 73, 268-271 (1994).

39. Ludwig, S., Nalbach, P., Rosenberg, D. \& Osheroff, D. Dynamics of the destruction and rebuilding of a dipole gap in glasses. Phys. Rev. Lett. 90, 105501 (2003).

40. Martinis, J., Nam, S., Aumentado, J., Lang, K. \& Urbina, C. Decoherence of a superconducting qubit due to bias noise. Phys. Rev. B 67, 094510 (2003).

41. de Visser, P. J. et al. Number fluctuations of sparse quasiparticles in a superconductor. Phys. Rev. Lett. 106, 167004 (2011).

42. Earnest, C. T. et al. Substrate surface engineering for high-quality silicon/aluminum superconducting resonators. Supercond. Sci. Technol. 31, 125013 (2018).

43. Rigetti, $C$. et al. Superconducting qubit in a waveguide cavity with a coherence time approaching 0.1 ms. Phys. Rev. B 86, 100506(R) (2012).

44. Barends, R. et al. Minimizing quasiparticle generation from stray infrared light in superconducting quantum circuits. Appl. Phys. Lett. 99, 113507 (2011).

45. Grünhaupt, L. et al. Loss mechanisms and quasiparticle dynamics in superconducting microwave resonators made of thin-film granular aluminum. Phys. Rev. Lett. 121, 117001 (2018).

46. Nugroho, C. D., Orlyanchik, V. \& Van Harlingen, D. J. Low frequency resistance and critical current fluctuations in al-based josephson junctions. Appl. Phys. Lett. 102, 142602 (2013).

47. Koch, J. et al. Charge-insensitive qubit design derived from the Cooper pair box. Phys. Rev. A 76, 042319 (2007).

48. O'Malley, P. J. J. et al. Qubit metrology of ultralow phase noise using randomized benchmarking. Phys. Rev. Appl. 3, 044009 (2015).

49. Schlör, S. et al. Correlating decoherence in transmon qubits: low frequency noise by single fluctuators. Preprint at arXiv:1901.05352 (2019).

50. Hong, S. et al. Demonstration of a parametrically-activated entangling gate protected from flux noise. Preprint at arXiv:1901.08035 (2019).

51. Gladchenko, S. et al. Superconducting nanocircuits for topologically protected qubits. Nat. Phys. 5, 48-53 (2009).

52. Burnett, J., Bengtsson, A., Niepce, D. \& Bylander, J. Noise and loss of superconducting aluminium resonators at single photon energies. J. Phys. Conf. Ser. 969, 012131 (2018).

53. Pop, I. M. et al. Coherent suppression of electromagnetic dissipation due to superconducting quasiparticles. Nature 508, 369-372 (2014).

54. Van Vliet, C. M. \& Handel, P. H. A new transform theorem for stochastic processes with special application to counting statistics. Phys. A 113, 261-276 (1982).

Open Access This article is licensed under a Creative Commons Attribution 4.0 International License, which permits use, sharing, adaptation, distribution and reproduction in any medium or format, as long as you give appropriate credit to the original author(s) and the source, provide a link to the Creative Commons license, and indicate if changes were made. The images or other third party material in this article are included in the article's Creative Commons license, unless indicated otherwise in a credit line to the material. If material is not included in the article's Creative Commons license and your intended use is not permitted by statutory regulation or exceeds the permitted use, you will need to obtain permission directly from the copyright holder. To view a copy of this license, visit http://creativecommons. org/licenses/by/4.0/.

(c) The Author(s) 2019 